\title{
Humanes Mikrobiom - Wie Mensch und Mikrobe zusammenwirken
}

Die neuere FORSCHUNG zeigt, welche Bedeutung Keime für uns haben. Jürgen Schulze

DIE PHYLOGENETISCHE und ontogenetische Entwicklung aller mehrzelligen Organismen findet unabdingbar in Koevolution mit Mikroorganismen statt. Im Laufe der Evolution hat der Mensch mit Mikroben auf seinen äußeren und inneren Oberflächen koexistierende Lebensgemeinschaften gebildet. Die Gesamtheit der dort nachweisbaren Mikroorganismen stellt die Mikrobiota des Menschen (synonym für Körperflora) dar. Man findet sie

- auf den äußeren Oberflächen:

Haut, Haaren, Nägeln

- auf den Kontaktzonen: Konjunktiva, äußeres Ohr, Urethra, äußerer Bereich der Vagina, Zervix

- im Respirationstrakt: Nase, Mund, Rachen

- im Verdauungstrakt: Magen, Dünndarm, Dickdarm

An jedem Standort bilden sich eigenständige Lebensgemeinschaften zwischen Mensch und Mikroben heraus (Mikrobiozönosen). Besonders intensiv werden das Vorkommen und die Wirkungsweise von Mikroorganismen im Verdauungstrakt untersucht. Die Summe der im MagenDarm-Trakt nachweisbaren lebenden Mikroorganismen (ca.1014 KBE = Koloniebildende Einheiten) wird als Mikrobiota des Gastrointestinaltrakts (synonym für Darmflora) bezeichnet.

\section{Quantensprünge in der mikrobiellen Analytik}

Bis in die 1980er-Jahre identifizierten Fachleute und Speziallabors die Mikroor-

\author{
KURZ GEFASST \\ 1 Keime galten lange einzig als potenzielle Krankheitserreger, die es zu \\ vernichten galt. Dabei sind manche, im Darm und auf anderen Kör- \\ peroberflächen angesiedelte, wichtig für die Gesundheit. \\ 2 Der Mensch lebt in einer Lebensgemeinschaft mit 100 Billionen Bak- \\ 2 terien. Diese bringen 150-mal mehr Gene mit, als der Mensch in sei- \\ nen eigenen Körperzellen hat. \\ 3 Die Mikrobiota trägt zur Steuerung physiologischer Vorgänge und \\ 3 Aufrechterhaltung der Gesundheit bei. Sie agiert als Superorgan in \\ Kooperation mit dem Makroorganismus.
}

ganismen sowohl qualitativ als auch quantitativ ausschließlich mittels kultureller, biochemischer, physikochemischer und mikroskopischer (inkl. elektronenmikroskopischer) Techniken. Die Entwicklung molekularbiologischer Analyseverfahren, v. a. die Einführung der DNA-Sequenzierung, hat die humanmedizinische mikroökologische Forschung revolutioniert. Mit den sehr zeitaufwändigen traditionellen Methoden war es über viele Jahrzehnte gelungen, durch Kultivierung auf geeigneten Nährböden etwa 450 verschiedene Arten von im Magen-DarmTrakt lebenden Mikroorganismen zu identifizieren. Heute sind bereits mehr als 1000 Mikroorganismen bekannt (s. u.), die sich anhand ihrer genetischen Determinanten als eigenständige Arten der Magen-Darm-Mikrobiota des Menschen differenzieren lassen. Hochrechnungen zufolge vermuten Experten bis zu etwa 10000 Mikroorganismen-Arten in unserer Mund-Magen-Darm-Mikrobiota. Obwohl ca. $80 \%$ davon unbekannte und nicht kultivierbare Bakterien sein dürften, ist es durch Abgleich von Sequenzähnlichkeiten mit bekannten Genen möglich, Informationen über Enzyme, Stoffwechselleistungen und ihre Bedeutung innerhalb des mikroökologischen Systems zu erhalten. Immer ausgeklügeltere Sequenzierungsmethoden, gepaart mit einer HochleistungsRechentechnik, die den Zeitbedarf für die Identifikation des genetischen Materials extrem verkürzt, lassen die Forschung dazu boomen.

\section{Das Mikrobiom und sein Metagenom}

Nachdem im Jahr 2001 das humane Genom (Gesamtheit aller Gene der körpereigenen Zellen) mit etwa 22000 Genen entschlüsselt worden war, wurden 2007/2008 etwa zeitgleich in den USA das Human Microbiom Project (HMP) und in Europa das Konsortium für Metagenomics of the Human intestinal tract (MetaHit) initiiert. Es 


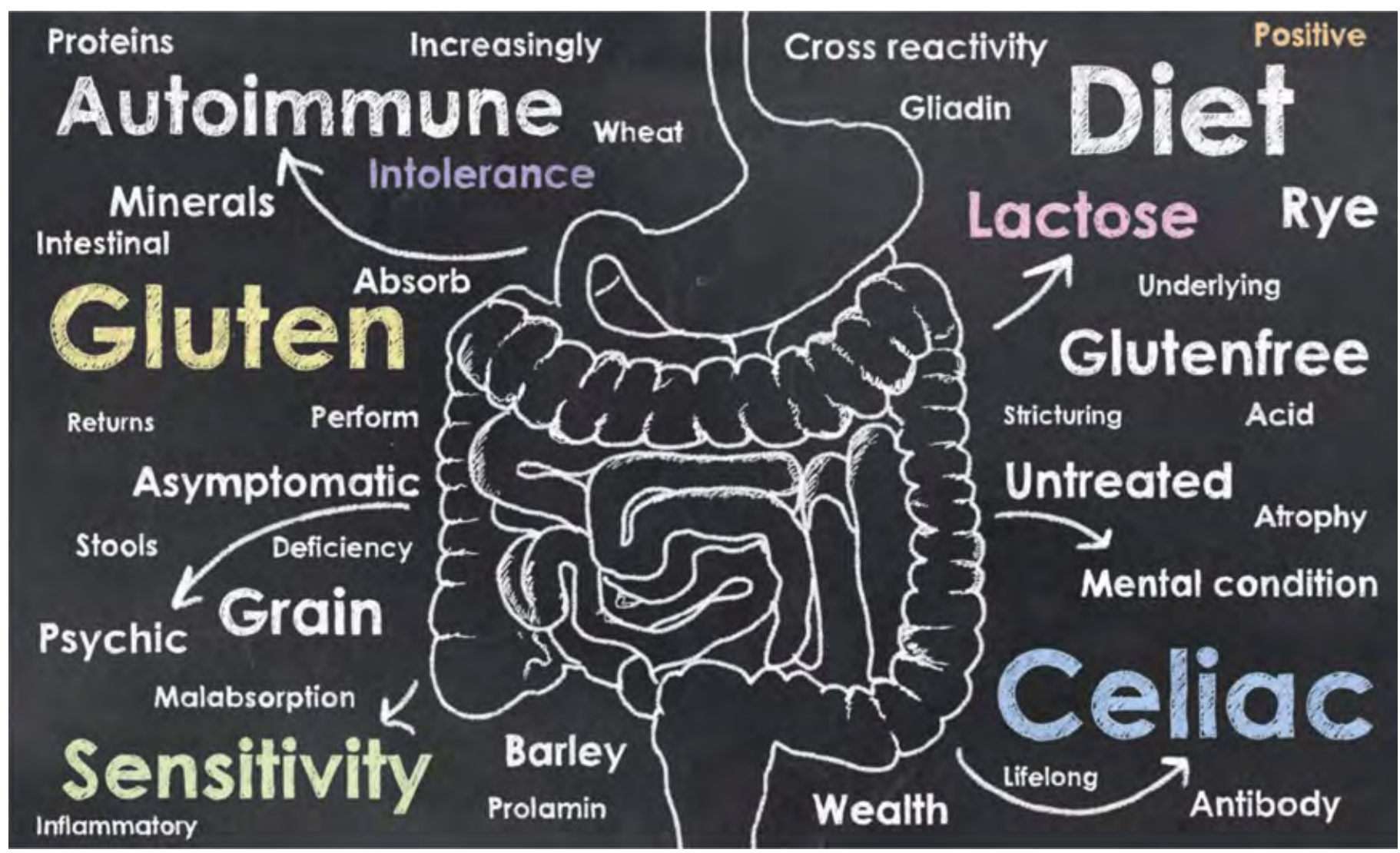

Abb. 1 Das gastrointestinale Mikrobiom mit seiner Vielzahl an Mikroorganismen greift auf unterschiedlichsten Ebenen in unseren Stoffwechsel ein. Es unterhält nicht nur Nahrungsmittelunverträglichkeiten, sondern ist, wie aktuelle Forschungen beweisen, auch an der Entstehung zahlreicher intestinaler, metabolischer und psychischer Erkrankungen beteiligt. Foto: @ Fotolia/T. L. Furrer

war der US-amerikanische Nobelpreisträger und Molekularbiologe Joshua Lederberg, der bereits 2001 forderte, das Genom des Menschen nicht losgelöst von den Genen seiner Mikrobiota zu betrachten.

Mikroorganismen und ihre Gene Lederberg prägte den Begriff „Mikrobiom“ für den „anderen Teil des Menschen“, nämlich für „seine“ Mikroorganismen mit ihren genetischen Ausstattungen und ihren Beziehungen (z. B. Stoffwechsel, Immunmodulation etc.) zum jeweiligen Standort in unserem Körper. Damit führte er die ganzheitliche Betrachtungsweise, die seit langem Kernstück der naturheilkundlichen Forschung und Praxis ist, auch in die schulmedizinischen Fachgebiete mit mikroökologischem Hintergrund ein. Die erste Komplettanalyse des kollektiven Genoms aller Mikroorganismen auf und in unserem Körper, des Metagenoms, ergab 3300000 Gene [11]. Nach neueren Angaben, bei denen neben Bakterien und Pilzen auch Viren einbezogen sind, sollen es ca. 8000000 Gene sein, d. h. auf 1 humanes Gen kommen mindestens 150, möglicherweise sogar 360 mikrobielle Gene. Diese enorm hohe Gen-Dichte im Mikrobiom ermöglicht in erheblichem Maße den Austausch von Gen-Substanz, mithin auch den Austausch funktioneller Eigenschaften [13].

\section{- INFORMATION}

\section{Was ist was?}

- Mikrobiota: Gesamtheit aller Mikroorganismen eines definierten Standorts

- Metagenom: Gesamtheit der Gene und Genome aller Mikroorganismen eines definierten Standorts

- Mikroökologie: Teilgebiet der Biologie, das sich mit den Beziehungen zwischen und innerhalb der Mikroorganismen (Lebensgemeinschaften) und der sie umgebenden Außenwelt (Helmut Haenel, 1960) befasst
Mikrobiom als Multitalent und Superorgan

Das humane Mikrobiom trägt im großen Umfang zur Steuerung physiologischer Vorgänge und Aufrechterhaltung der Gesundheit bei. Es sorgt für den Aufbau, die Stabilität und partiell für die Reparatur einer unspezifischen Barriere zur Abwehr 
von Infektionskeimen. Gleichzeitig nimmt es Einfluss auf die Entwicklung und Aktivität des darmassoziierten Immunsystems. Mit unüberschaubaren metabolischen Aktivitäten (etwa 30 \% der Metaboliten im Blut sind mikrobiellen Ursprungs) ist die Mikrobiota in den humanen Metabolismus integriert. Beispielhaft sei erwähnt, dass sich das Mikrobiom am Abbau komplexer Polymere aus der Nahrung beteiligt und dem Wirt zusätzlich Energie, aber auch Vitamine $\left(\mathrm{B}_{12}\right.$, Folsäure, Biotin, Vitamin $\mathrm{K}_{2}$ ) zur Verfügung stellt. Mikrobiell gebildete kurzkettige Karbonsäuren fördern die Durchblutung der Darmschleimhaut (Acetat), haben einen positiven Effekt auf den Cholesterolstoffwechsel der Leber (Propionat), stabilisieren die Funktion der Kolonozyten bei gleichzeitiger Verminderung der Entzündungsaktivität (Butyrat) und reduzieren über die pH-Neutralisation im Dickdarm die Ammoniak-Last (Laktat). Katalasen von Laktobazillen wirken über die Reduktion oxidativer Radikale detoxifizierend und ermöglichen die Entgiftung von toxischen oder (pro)kanzerogenen Stoffen exogenen oder endogenen Ursprungs bereits im Darm, dessen Milieu im Wesentlichen von der Funktion des Mikrobioms bestimmt wird [14].

\section{Ein neues Zuhause im Baum des Lebens}

Das wachsende Bedürfnis, Verwandtschaftsgrade zwischen einzelnen Arten nicht nur an phänotypischen, also vom Erscheinungsbild geprägten, sondern auch an genotypischen Merkmalen aufzustellen, hat in den letzten 50 Jahren zur Revision der Systematik geführt. Auf Basis von rRNA-Genen ließ sich der gesamte „phylogenetische Baum des Lebens“ auf 3 Domänen reduzieren, nämlich die Archaeen (früher Urbakterien), Bakterien (beides sind Prokaryonten) und Eukaryonten (alle Lebewesen mit einem Zellkern). Vor allem die Möglichkeit der Gensequenzierung hat die Grundlage für neu entdeckte genetisch determinierte Verwandtschaften und somit für ein völlig neues Arrangement des taxonomischen Systems der Mikroorganismen geliefert.

Die Domäne der Bakterien umfasst zurzeit 29 Phyla (Stämme; die verschiede- nen Bakteriengattungen, aus denen sich nach heutigem Verständnis die Mikrobiota des Gastrointestinaltrakts rekrutiert, finden sich in Tab. 1).

Im Wesentlichen sind es nur 4 Phyla (Firmicutes, Bacteroidetes, Actinobacteria, Protobacteria), zu denen Hunderte von Bakterienarten gehören und die mit > 95 \% aller mikrobiellen Zellen das Gros der Darm-Mikrobiota bilden. Den restlichen Anteil (< $5 \%$ ) stellen Mikroorganismen-Arten, die aus den Phyla Fusobacteria, Verrucomicrobia, Euryarcheota und Ascomycota stammen [4].

Bei Analysen zur kompletten fäkalen Mikrobiota, die mittels DNA-Sequenzierung in Proben von 124 Europäern durchgeführt wurden, fanden sich ca. 10001150 unterschiedliche, kultivierbare und nicht kultivierbare Bakterienarten [11]. Mindestens 160 der identifizierten Arten wurden bei jedem Probanden gefunden. Trotz individueller Unterschiede in der Artenvielfalt scheint jede individuelle Mikrobiota mit einem „Kern-Mikrobiom“ ausgestattet zu sein, das Störungen des Systems besser übersteht.

\section{Stamm oder keiner?}

Bei Bakterien und Archaeen ist der Begriff „Phylum“ (deutsch: Stamm) eine hierarchische Rangstufe unterhalb der Domäne. Keine taxonomische Rangstufe, sondern eine Bezeichnung für eine Abstammungslinie (Klon) ist die in der Praxis häufig verwendete Bezeichnung „Bakterienstamm“ (engl.: bacterial strain) innerhalb einer Art. So wird z. B. mit der Bezeichnung E. coli Stamm Nissle 1917 eine Abgrenzung zu anderen Vertretern derselben Art E. coli festgelegt.

\section{Das Essen definiert unsere Mikrobiota}

Ein weiteres überraschendes Ergebnis war, dass die Zusammensetzung der Mikrobengemeinschaft im Darm bestimmten Ordnungsprinzipien zu unterliegen scheint. Demnach ist die Darm-Mikrobiota (fast) jedes Menschen 3 Clustern mit typischer Bakteriengruppen-Dominanz, den sog. „Enterotypen“, zuzuordnen. Diese wurden nach der jeweils dominierenden Gattung benannt (s. Kasten). Die Zuordnung zu ei-
INFORMATION

\section{Dominierende Mikroben- gemeinschaften des Darmes}

Enterotyp 1 - Bacteroides-Dominanz

- Besonderheit: ist spezialisiert auf den Abbau komplexer Kohlenhydrate und (tierischem) Eiweiß durch Fermentation sowie auf die Synthese von Biotin (Vitamin $B_{5}$ ), Vitamin $B_{2}$, Panthothensäure (Vitamin $B_{7}$ ) und Ascorbinsäure (Vitamin C)

- Bezug zur Ernährung: positive Korrelation zum Fleischverzehr

Enterotyp 2 - Prevotella-Dominanz

- Besonderheit: ist spezialisiert auf den Abbau von Zucker-Protein-Komplexen aus Muzinen, Synthese von Folsäure und Vita$\min B_{1}$

- Bezug zur Ernährung: positive Korrelation zum Vegetarismus

Enterotyp 3 - Ruminococcus-Dominanz

- Besonderheit: ist spezialisiert auf den Abbau von Zellulose, Muzinen und desaminierten Kohlenstoffgerüsten (Zucker), Alkoholen (unter allen untersuchten Proben die am häufigsten gefundene Konstellation)

- Bezug zur Ernährung: keine klare Korrelation zu einer Ernährungsform, evtl. Mischkost

nem der 3 Enterotypen scheint unabhängig von Nationalität, Alter, Geschlecht und Body-Mass-Index zu sein. Allerdings besteht eine enge Assoziation zum LangzeitErnährungsverhalten [1], was den seit Langem vermuteten, aber schwer nachweisbaren Einfluss der Ernährung auf die Darm-Mikrobiota nahelegt.

Die Annahme, dass der Einfluss auf die Mikrobiota nur über ein Langzeitdiätregime funktioniert, ist durch aktuelle Untersuchungsergebnisse infrage gestellt [5]. In einem Pilotprojekt wurde 10 Mischköstlern (21-33 Jahre alt) entweder eine Diät tierischer Herkunft (Fleisch, Eier, Käse) oder eine vegetarische Diät (Getreide, Hülsenfrüchte, Gemüse, Früchte) verabfolgt. Unter der tierischen Kost wurde bereits nach einem Tag die Anzahl der vorherrschenden galletoleranten Mikroorganismen Alistipe, Bilophila und Bacteroides 
- TABELLE 1

\section{Auswahl der für die Mikrobiota des Gastrointestinaltrakts relevanten Gattungen und die robuste} taxonomische Zuordnung zum „phylogenetischen Baum des Lebens“ [modifiziert nach 4]

\begin{tabular}{|c|c|c|c|}
\hline Archaeen & Euryarchaeota & Methanobacteriales & Methanobrevibacter \\
\hline \multirow[t]{7}{*}{ Bakterien } & Actinobacteria & Bifidobacteriales & Bifidobacterium \\
\hline & Bacteroidetes & Bacteroidales & $\begin{array}{l}\text { Bacteroides } \\
\text { Prevotella } \\
\text { + weitere } 2 \text { Gattungen }\end{array}$ \\
\hline & & Erysipelotrichales & $\begin{array}{l}\text { Coprobacillus } \\
\text { Holdemania } \\
\text { Catenibacterium }\end{array}$ \\
\hline & & Selenomonadales & $\begin{array}{l}\text { Veillonella } \\
\text { + weitere } 28 \text { Gattungen }\end{array}$ \\
\hline & Fusobacteria & Fusobacteriales & Fusobacterium \\
\hline & Proteobacteria & Enterobacteriales & $\begin{array}{l}\text { Escherichia } \\
\text { Enterobacter }\end{array}$ \\
\hline & Verrucomicrobia & Verrucrobiales & Akkermannsia \\
\hline Eukaryonten & Ascomycota & Saccharomycetales & Candida \\
\hline
\end{tabular}


weiter erhöht und der Gehalt an Firmicuten (Roseburia, Eubacterium und Ruminicoccus), die v. a. pflanzliche Polysaccharide degradieren, vermindert. Unter dem Einfluss der Ernährung änderte sich nicht nur die qualitative und quantitative $\mathrm{Zu}$ - sammensetzung der Mikrobiota, sondern auch das Mikrobiom mit dem Bezug zu möglichen gesundheitlichen Konsequenzen. Das fäkale Gallensäureprofil zeigte eine Erhöhung der Desoxycholsäurekonzentration, die mit der Entstehung von Le-

- TABELLE 2

\section{Ursachen für strukturelle und metabolische Veränderungen im intestinalen Mikrobiom [modifiziert nach 14]}

\begin{tabular}{|c|c|c|}
\hline \multicolumn{2}{|l|}{ Ursachen } & \multirow{2}{*}{$\begin{array}{l}\text { Beispiele } \\
\text { Infektionen } \\
\text { Toxine } \\
\text { Allergene }\end{array}$} \\
\hline \multirow{3}{*}{ Umwelt-Noxen } & biologische & \\
\hline & chemische & $\begin{array}{l}\text { Schwermetalle } \\
\text { Herbizide } \\
\text { Fungizide } \\
\text { Insektizide } \\
\text { Desinfektionsmittel }\end{array}$ \\
\hline & physikalische & energiereiche Strahlung \\
\hline \multirow[t]{2}{*}{ Therapeutische Maßnahmen } & $\begin{array}{l}\text { Medikamente } \\
\text { Strahlen }\end{array}$ & $\begin{array}{l}\text { Antibiotikatherapie } \\
\text { Chemotherapie } \\
\text { weitere Medikamente } \\
\text { Strahlentherapie } \\
\text { Röntgenstrahlen }\end{array}$ \\
\hline & chirurgische Eingriffe & $\begin{array}{l}\text { Teilresektionen } \\
\text { blinde Schlingen }\end{array}$ \\
\hline \multirow{3}{*}{ Erkrankungen } & strukturelle & $\begin{array}{l}\text { Divertikel } \\
\text { Stenosen } \\
\text { Karzinome }\end{array}$ \\
\hline & systemische & $\begin{array}{l}\text { metabolische, z. B. Diabetes } \\
\text { Immundefekte }\end{array}$ \\
\hline & psychosoziale & $\begin{array}{l}\text { psychische Belastungen } \\
\text { emotionale Stressoren }\end{array}$ \\
\hline \multirow[b]{2}{*}{ Nahrung und Ernährung } & Ernährungsverhalten & $\begin{array}{l}\text { Fehlernährung (zu fett, zu eiweißreich) } \\
\text { extremes Fasten } \\
\text { totale parenterale Ernährung (TEP) }\end{array}$ \\
\hline & Lebensmittelzusatzstoffe & $\begin{array}{l}\text { Farbstoffe } \\
\text { Geschmackstoffe } \\
\text { Süßstoffe } \\
\text { Konservierungsstoffe }\end{array}$ \\
\hline
\end{tabular}




\section{Entwicklung der Darm-Mikrobiota}

- Der erste Kontakt mit Kompartimenten des mütterlichen Mikrobioms findet in utero statt.

- Der Siedlungsprozess setzt sich unter der Geburt fort.

- In der Folge bilden sich standortspezifische Partnerschaften (Mikrobiozönosen) zwischen Makro- und Mikroorganismen heraus.

- Speziesspezifische Gesetzmäßigkeiten und umweltspezifische Zufälligkeiten (z. B. Herkunft der Mikroorganismen, Ernährung, Medikamente, Fremdstoffe) bestimmen die mikroökologischen Beziehungen und den Charakter des Mikrobioms.

stillt werden, rekrutiert sich die Mikrobiota über einen zusätzlichen Weg aus dem Bakterienpool der Mutter. Via Muttermilch erfolgt der Transfer v. a. obligat anaerob lebender Darmbakterien (z. B. Bifidobakterien) auf den Säugling, was in den letzten Jahren sowohl über Gen-Sequenzierung als auch kulturell mehrfach nachgewiesen worden ist. Zwar ist der diskutierte DarmBrustdrüsen-Weg noch hypothetisch [10], aber mit mehr als 600 nachgewiesenen Bakterienarten enthält die Muttermilch viel mehr Mikroorganismen als erwartet [8]. Die Umstellung vom fakultativen zum strikt anaeroben Mikrobiom erfolgt demnach viel früher als ursprünglich angenommen [9]. Muttermilch liefert außerdem verschiedene Galakto-Oligosaccharide und Aminozucker, die als „Bakterienfutter“ (Präbiotika) für das Keimzahlwachstum und den Stoffwechsel v. a von Bifidobakterien wertvoll sind. Bereits zu Beginn des Lebens übernimmt die Ernährung eine prägende Rolle bei der Genese des individuellen intestinalen Mikrobioms. Säuglinge, die anstelle der Muttermilch eine FormulaMilch erhalten, entwickeln in den ersten Wochen und Monaten ihres Lebens eher eine fäulnisbetonte Darm-Mikrobiota als die säurebetonte Bifidobakterien-Mikrobiota. Die wünschenswerte Artenvielfalt der Muttermilch ist jedoch eingeschränkt, wenn die Mütter übergewichtig sind, mit Kaiserschnitt entbunden haben und/oder mit Antibiotika behandelt wurden.

\section{Was schadet der Mikrobiota - und uns?}

Die ersten fundierten Erkenntnisse zur Bedeutung der intestinalen Mikrobiota für die Erhaltung der Gesundheit oder
Entstehung von Krankheiten stammen aus Untersuchungen gegen Ende des 19. Jahrhunderts und sind mit Namen hervorragender Forscherpersönlichkeiten wie Louis Pasteur, Theodor Escherich, Ilja Metschnikoff, Robert Koch u. a. verbunden. Als Ursachen für Störungen der Mikroflora des Magen-Darm-Trakts wurden seinerzeit ausschließlich Infektionen mit pathogenen Mikroorganismen (zunächst nur Bakterien, später auch Pilze und Viren) dingfest gemacht. Der Freiburger Arzt und Hygieniker Alfred Nissle beschrieb erstmalig 1916 den „Coli-Antagonismus“ und stellte „die Grundlage einer neuen ursächlichen Bekämpfung der pathologischen Darmflora“ mit Mikroorganismen vor.

\section{Antibiotika und weitere Arzneimittel}

Die meisten Nebenwirkungen von Antibiotikatherapien äußern sich als gastroenterologische Beschwerden (am häufigsten Antibiotikaassoziierte Diarrhö), weil nicht nur Pathogene eliminiert sondern auch physiologische Mikroorganismen der Darm-Mikrobiota geschädigt werden. Weltweit sind ca. 8000 antibiotisch wirksame Substanzen im Einsatz, die Jahresproduktion beläuft sich auf ca.100 000 Tonnen. Antibiotika sind für das gastrointestinale Mikrobiom „Feind Nr. 1“. Weitere Arzneimittel, wie Medikamente zur Behandlung von Krebs, Schmerzmittel, Abführmittel oder Kortison, schädigen die Mikrobiota in unterschiedlichem Maße, ebenso wie Strahlen, Umweltgifte und Lebensmittelzusatzstoffe.

\section{Erkrankungen}

Ebenso nehmen diverse Erkrankungen Einfluss auf verschiedene Funktionen des Mikrobioms (s. Tab. 2). 


\section{Einfalt oder Vielfalt?}

In der Vergangenheit wurde bei der Suche nach krankheitsbedingten Veränderungen in der Mikrobiota das Hauptaugenmerk auf einzelne Mikroorganismen (Pathogene oder sog. Leitkeime) gelegt, die am Krankheitsgeschehen ursächlich oder begleitend beteiligt sind. Alle für dieses Ziel optimierten mikrobiologisch-kulturellen Methoden erfüllen auch heute noch ihren Zweck. Allerdings ist den monolytischen Verfahren mit den neuen hochparallelen Sequenzierungstechniken und 16s-rDNA-basierten Identifikationsverfahren eine Untersuchungsmaschinerie zur Seite gestellt worden, die in kürzester Zeit nach Probenahme eine Flut von zusätzlichen Informationen erzeugt. Diese geben Auskunft über die funktionelle Kapazität aller beteiligten Mikroorganismen und vermitteln anhand der Identifikation enormer Mengen bakterieller Signaturen einen Eindruck über die Vielfältigkeit („diversity“) der Mikrobiota.

Zusammengefasst ergibt sich, dass eine große Vielfalt an Mikroorganismen den besten Schutz vor Störfaktoren bietet und ein „gesundes“ Mikrobiom charakterisiert. Umgekehrt korreliert eine Verarmung der mikrobiellen Vielfalt des Mikrobioms mit Krankheit.

Merke: Auch mit zunehmendem Lebensalter nimmt der Artenreichtum im Mikrobiom ab.

\section{Neues Krankheitsverständnis, neue Behandlungsoptionen}

Es ist bekannt, dass Störungen im Gleichgewicht der gastrointestinalen Mikrobiota sowohl zu Erkrankungen im MagenDarm-Trakt als auch zu extraintestinalen Erkrankungen führen können.

\section{Gastrointestinale Erkrankungen}

Enge Korrelationen zu einem gestörten Mikrobiom bestehen bei der Antibiotikaassoziierten Diarrhö, bei infektiösen Durchfällen und Darmmykosen, beim bakteriellen Überwucherungssyndrom des Dünndarms (SIBO), bei chronischen Darmerkrankungen (CED: Morbus Crohn, Colitis ulcerosa, mikroskopische Kolitis), bei Nahrungsmittelunverträglichkeiten und beim postinfektiösen Reizdarmsyndrom (PI-RDS).
Schwache Korrelationen bestehen zur chronischen Obstipation, weiteren RDSEntitäten und zum kolorektalen Karzinom.

\section{Extraintestinale Erkrankungen}

Ein gestörtes intestinales Mikrobiom findet sich aber auch bei zahlreichen extraintestinalen Erkrankungen: bei rekurrierenden Harnwegsinfektionen, rezidivierenden Vulvovaginalmykosen, enterogenen Arthritiden, reaktiven Arthritiden und Spondylarthritiden; ferner bei chronischen und allergischen Erkrankungen der Haut und Atemwege (Neurodermitis, Psoriasis, Urtikaria, Asthma) und bei der hepatischen Enzephalopathie.

Neue Einsichten in die Zusammenhänge zwischen Mikrobiom und Krankheiten hat das umfangreiche Datenmaterial über die Vielfältigkeit der Mikrobiota inkl. des möglichen Genaustauschs geliefert. So ist sicher, dass durch Stress verursachte Erkrankungen, Angst und Depressionen, Multiple Sklerose und Autismus über das Mikrobiom getriggert werden.

\section{Behandlungsoptionen}

Die funktionelle Kapazität des gesamten Mikrobioms eröffnet neue Wege zur Therapie z. B. des metabolischen Syndroms (Adipositas, Diabetes mellitus, Fettstoffwechselstörungen, Atherosklerose). Die meisten optimistischen Ergebnisse zu diesen Beziehungen stammen zwar aus Tierversuchen. Nach und nach werden sie jedoch für den Menschen verifiziert. So ist bei Adipösen der relative Gehalt an Mikroorganismen des Phylums Bakteroidetes niedriger und der des Phylums Firmicutes höher als bei Normalgewichtigen. Im Mikrobiom Normalgewichtiger, die eine hochkalorische Diät erhielten, änderte sich rasch das Verhältnis dieser beiden Phyla zueinander: Der Anteil der Firmicuts stieg, der der Bacteroidetes sank um $20 \%$. Gegenläufig veränderte sich das Verhältnis beider Phyla bei Adipösen, die sich über ein Jahr mit einer energiereduzierten Diät ernährten. Die Transplantation einer Mikrobiota von morbidadipösen Patienten in normalgewichtige Mäuse löste bei diesen Adipositas aus [12]. Im Mikrobiom Adipöser finden sich höhere Aktivitäten von Glycosidhydrolasen und Pyruvat-Formiat-Lyasen, was auf eine erhöhte Spaltungs- und Verwertungsaktivität von Bal- laststoffen hinweist. Adipöse erhalten also mehr Energie aus angeblich nicht verwertbaren Ballaststoffen als Normalgewichtige [15].

\section{Therapie mit Bakterien}

Das Prinzip, eine gestörte Darm-Mikrobiota durch die Zufuhr lebender physiologischer Mikroorganismen wieder ins Gleichgewicht zu bringen, hat bereits eine über 100-jährige Geschichte. Mutaflor ${ }^{\circledR}$ ist das älteste als Arzneimittel zugelassene Bakterienpräparat mit dem probiotischen E.-coli-Stamm Nissle 1917. Die Fortschritte der Antibiotika-Forschung in der 2. Hälfte des vergangenen Jahrhunderts ließen allerdings den therapeutischen Einsatz von Mikroorganismen in den Hintergrund geraten. Erst das vermehrte Auftreten gegen Antibiotika resistenter Infektionskeime hat in den 1990erJahren zu einer Wiederbelebung der probiotischen Therapie geführt [14]. Jährlich erscheinen weit über 1000 internationale Fachpublikationen zu Wirkmechanismen und zur Wirksamkeit von Probiotika. Der Aufschwung, den die frühere DarmfloraForschung durch die neuen Möglichkeiten der Mikrobiom-Forschung erhält, ist natürlich gekoppelt mit dem Wunsch nach effizienten, möglichst personalisierten therapeutischen Strategien.

Ausgerechnet eine antiquierte Methode, die aufgeführt ist im Handbuch für medizinische Notfälle des chinesischen Arztes Ge Hong, der in der Dongjin-Dynastie des 4. Jahrhunderts lebte, und längst totgesagt ist - nämlich die Transplantation von Fäkalkeimen - genießt eine Renaissance [2]. Die Clostridium-difficile-Diarrhö, die häufigste Form der nosokomialen infektiösen Enteritis, wird standardmäßig mit den Antibiotika Vancomycin und Metronidazol therapiert. Rund ein Viertel der Patienten erleidet jedoch ein Rezidiv, was für ältere und multimorbide Patienten lebensgefährlich sein kann. Im Verlauf der letzten 50 Jahre sind mehrere Fallbeispiele mit erfolgreicher Therapie durch Stuhlübertragung berichtet worden. In einer kürzlich publizierten offenen, randomisierten und kontrollierten Studie lag die Heilungsrate nach Stuhltransplantation bei $93 \%(15 / 16)$, bei 2 Kontrollgruppen nach Vancomycintherapie bei $31 \%$ (4/13), bzw. $23 \%$ (3/13) [16]. Zurzeit wird weltweit versucht, die Me- 
thode zu standardisieren, um sie auch für weitere Indikationen einsetzen zu können. Ein standardisiertes, möglicherweise personalisiertes Mikrobengemisch mit hoher Diversität könnte der Schlüssel zum Erfolg sein.

\section{Dieser Artikel ist online zu finden:} http://dx.doi.org/10.1055/s-0034-1396949

\section{Literatur}

[1] Arumugam M, Raes ], Pelletier E, Le Paslier D, Yamada T, Mende DR et al. Enterotypes of the human gut microbiome. Nature 2011; 473: $174-180$

[2] Baumgart DC. Bedeutung des Mikrobioms heute. Current Congress - Viszeralmedizin 2014: 20-21. Letzter Aufruf 04.11.2014 https://www.thieme.de/statics/dokumente/ thieme/final/de/dokumente/zw_current-congress/CC_DGVS_2014_Leipzig.pdf

[3] Bianconi E, Piovesan A, Facchin F, Beraudi A, Casadei R, Frabetti $F$ et al. An estimation of the number of cells in the human body. Ann Hum Biol 2013; 40: 463-471

[4] Blaut M. Ecology and Physiology of the intestinal tract. Curr Topics Microbiol Immunol 2013; 358: 247-272

[5] David LA, Maurice CF, Carmodi RN, Gootenberg DB, Button JE, Wolfe BE et al. Diet rapidly and reproducibly alters human gut microbiome. Nature 2014; 505: 559-563

[6] Gosalbes M], Llop S, Valles Y, Moya A, Ballester $\mathrm{F}$, Francino MP. Meconium microbiota types dominated by lactic acid or enteric bacteria are differentially associated with maternal eczema and respiratory problems in infants. Clinical \& Experimental Allergy 2013; 43: 198-211

[7] Hehemann JH, Correc G, Barbeyron T, Helbert W, Czjzek M, Michel G. Transfer of carbohydrate-active enzymes from marine bacteria to Japanese gut microbiota. Nature 2010; 464: 908-912

[8] Hunt KM, Foster JA, Forney LJ, Schütte UME, Beck DL, Abdo Z, Fox LK et al. Characterization of the Diversity and Temporal Stabilty of Bacterial Communities in Human Milk. PLoS One 2011; 6: e21313. doi:10.1371

[9] Jost T, Lacroix C, Braegger CP, Chassard C. New insights in gut microbiota establishment in healthy breast fed neonates. PLoS One 2012; 7: e44595

[10] Jost T, Lacroix C, Braegger CP, Rochat F, Chassard C. Vertical mother-neonate transfer of maternal gut bacteria via breastfeeding. Environ Microbiol. 2014; 16: 2891 2904

[11] Qin J, Li R, Raes ], Arumugam M, Burgdorf KS, Manichanh C, Nielsen T et al. A human gut microbial gene catalogue established by metagenomic sequencing. Nature 2010; 464: 59-65
[12] Ridaura VK, Faith J], Rey FE, Cheng J, Duncan AE, Kau AL et al. Gut microbiota from twins discordant for obesity modulate metabolism in mice. Science 2013; 341: 1241214

[13] Schreiber S, Nikolaus S, Rosenstiel P. Mikrobiom und Ernährung -Therapie der Zukunft für chronisch entzündliche Darmerkrankungen? Internist 2014; 55 : 889-897

[14] Schulze J, Sonnenborn U, Ölschläger T, Kruis W. Probiotika - Mikroökologie, Mikrobiologie, Qualität, Sicherheit und gesundheitliche Effekte. Stuttgart: Hippokrates; 2008

[15] Turnbaugh PJ, Hamady M, Yatsunenko et al. A core gut microbiome in obese and lean twins. Nature 2009; 457: 480484

[16] Van Nood E, Vrieze A, Nieuwdorp M, Fuentes S, Zoetendal E et al. Duodenal infusion of donor feces for recurrant Clostridium difficele. N Engl j Med 2013; 368: 407-415

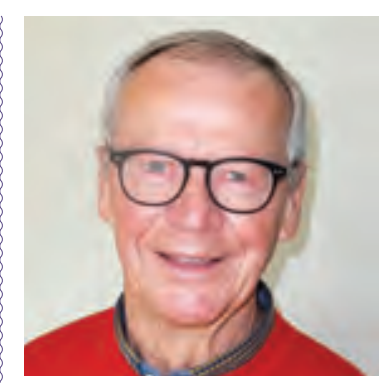

PD Dr. rer. nat. habil. Jürgen Schulze

Alice-Bloch-Str. 7

14558 Nuthetal

E-Mail: JuR.Schulze@t-online.de

Jürgen Schulze ist Diplom-Biologe und habilitierter Humanphysiologe mit folgenden Arbeitsbereichen: 25 Jahre leitende Forschungstätigkeit am Ernährungsinstitut in Potsdam-Rehbrücke (heute: Nuthetal) mit den Schwerpunkten gastrointestinale Mikrobiota und Mikroökologie, Säuglingsernährung, Ballaststoffe. Er war zudem 14 Jahre Bereichsleiter in der Pharmaindustrie (präklinische und klinische gastroenterologische Forschung), hatte einen Lehrauftrag für Pharmazeutische Mikrobiologie an der Rheinischen Friedrich-Wilhelms-Universität Bonn inne und ist Mitautor von Fachbüchern und über 130 wissenschaftlichen Publikationen u. a. zur Wirkweise und Wirksamkeit von Probiotika. 